\title{
A paradoxical cause of hypoxia and orthodeoxia in a stroke patient
}

\author{
P Soma, ${ }^{1}$ MB ChB, MSc (Clin Epi); D Joseph, ${ }^{2}$ MB ChB; $\mathbf{S}$ Ahmad, ${ }^{3}$ MBBS FCRAD (SA) Diploma in Neurointervention; \\ S Ellemdin, ${ }^{2} \mathrm{MB}$ ChB MMed (Int Med) \\ ${ }^{1}$ Department of Physiology, School of Medicine, Faculty of Health Sciences, University of Pretoria, South Africa \\ ${ }^{2}$ Department of Internal Medicine, School of Medicine, Faculty of Health Sciences, University of Pretoria and Steve Biko Academic Hospital, Pretoria, South Africa \\ ${ }^{3}$ Department of Radiology, School of Medicine, Faculty of Health Sciences, University of Pretoria and Steve Biko Academic Hospital, Pretoria, South Africa
}

Corresponding author: P Soma (prashilla.soma@up.ac.za)

The classic triad of dyspnoea on exertion, cyanosis and clubbing should alert the clinician to the possibility of pulmonary arteriovenous malformation (PAVM). Despite it being a rare condition, it is associated with significant clinical complications. A common feature is paradoxical emboli, with consequent complications such as brain abscess and stroke.

S Afr Respir J 2016;22(1):22-23. DOI: 10.7196/SARJ.2016.v22i1.42

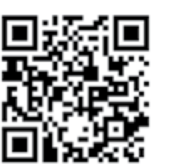

Pulmonary arteriovenous malformations (PAVMs) are best defined as low-resistance, high-flow abnormal vascular structures that often connect a pulmonary artery to a pulmonary vein, bypassing the normal pulmonary capillary bed and resulting in an intrapulmonary right-to-left shunt. ${ }^{[1]}$ The development of the right-left shunt results in hypoxia with shunting of micro emboli with central nervous system complications. ${ }^{[2]}$ PAVMs are most commonly congenital in nature with about $70 \%$ of cases associated with hereditary haemorrhagic telangiectasia (HHT). ${ }^{[2]}$ Physiologic consequences depend on the degree of right-to-left shunt and include hypoxaemia, dyspnoea, and cyanosis. ${ }^{[1]}$ Treatment options include surgery, embolisation therapy as well as hormonal and pharmacological interventions to prevent bleeding from arteriovenous malformations (AVMs). ${ }^{[2]}$ The triad of dyspnoea, cyanosis and clubbing is described as classic for PAVMs but may not always be present. ${ }^{[2]} \mathrm{We}$ describe a case of a young female presenting with a brain abscess and progressive neurological fallout. The presence of hypoxia and clubbing were clues to her underlying lung condition.

\section{Case report}

A 21-year-old woman presented to her local hospital with weakness of her right arm and leg as well as aphasia. Her symptoms started in September 2014 with impairment of speech followed by weakness over a period of 1 week. This was accompanied by a severe headache, nausea, vomiting, photophobia and blurred vision. Her past medical history was noncontributory. The patient assumed her unusual nails and blue discolouration of her lips and tongue were normal for her. She was burdened by dyspnoea on exertion while growing up. A computed tomography (CT) brain scan was done and she was discharged as having had a stroke after 2 weeks' inpatient care. With no improvement in her condition, she presented to our academic institution, Steve Biko Academic Hospital, Pretoria, in October 2014 with a complaint of chest pain.

On examination, she was afebrile, blood pressure was $97 / 77 \mathrm{mmHg}$ and heart rate was $90 \mathrm{bpm}$. She had both peripheral and central cyanosis with clubbing but insufficient criteria to meet the diagnosis of HHT (no history of epistaxis, absent telangiectasia and no family history). Her Glasgow coma scale (GCS) was 10/15, with abnormalities on the right side of the body including an upper motor neuron facial nerve palsy, $0 / 5$ power, reduced tone and positive clonus. In addition, there was hemisensory loss of all modalities. Her CT brain scan confirmed a left frontal lobe, thick-walled mass with surrounding vasogenic oedema suggestive of a brain abscess, and drainage thereof was performed.

Laboratory findings revealed a $\mathrm{PaO}_{2}$ $7.67 \mathrm{kPa}(57.5 \mathrm{mmHg}$ ), saturation of $91.4 \%$ on room air on admission. Supine and sitting blood gases varied with sitting $\mathrm{PaO}_{2} 5.87 \mathrm{kPa}$ (44.0 mmHg), saturation of $77.8 \%$ compared with supine $\mathrm{PaO}_{2} 6.91 \mathrm{kPa}(51.8 \mathrm{mmHg}$ ), saturation of $85.9 \%$. Despite the vascular abnormality located in the upper lobe, the patient presented with orthodeoxia. To exclude other causes of her stroke, antinuclear antibodies screen was performed and HIV serology, both of which were negative.
Her electrocardiogram and echocardiogram were normal. Her chest X-ray showed a large lobulated opacity in the right upper lung zone almost adjacent to the right hilum with some air bronchograms noted through the opacity. A CT scan of the chest confirmed the right upper lobe PAVM. This is likely causing a shunt that precipitated the septic embolism to the brain. The patient was referred to interventional radiology for AVM coiling where multiple coils were inserted as shown

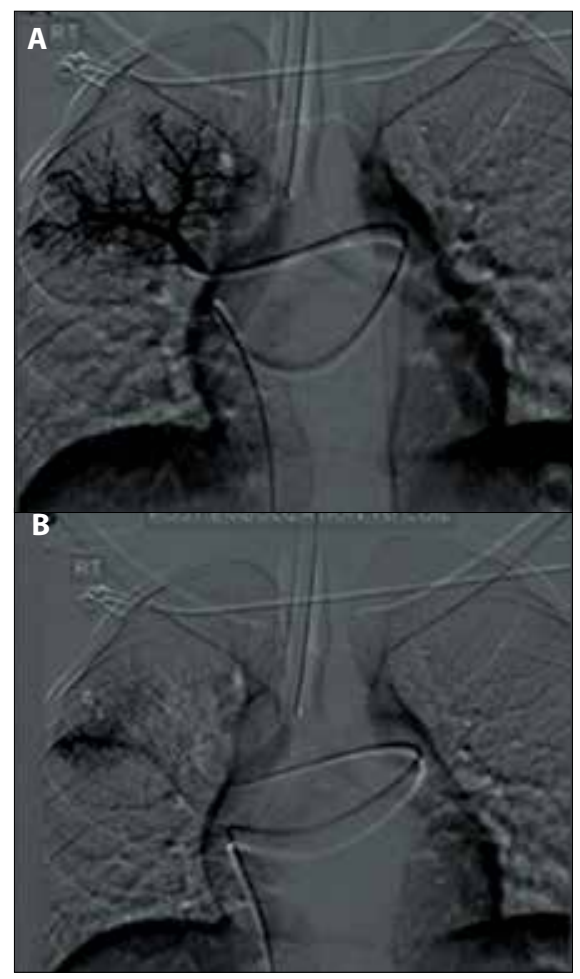

Fig. 1. (A) Pulmonary angiogram of right lung. (B) Embolisation of PAVM with few minor branches in AVM still visible. 
in Fig 1. Post intervention her blood gases improved to a $\mathrm{PaO}_{2} 12.22$ $\mathrm{kPa}(91.7 \mathrm{mmHg})$ and saturation of $96.8 \%$. However, upon followup her hypoxia had recurred, so on the 14 October 2015, the patient underwent a right and middle lobe lobectomy as the embolization resulted in partial improvement, the coils had opened up.

\section{Discussion}

PAVMs are abnormal communications between pulmonary veins and arteries ${ }^{[2]}$ Despite their rarity, mortality attributable to PAVMs is caused by serious neurological complications such as stroke, brain abscess, transient ischaemic attacks, migraine and seizures. ${ }^{[1,3]}$ The natural history of PAVMs is that they are inclined to increase in size and rarely regress spontaneously. Complications are more likely in the presence of hereditary haemorrhagic telangiectasia. ${ }^{[4]}$ Clinical presentation is a result of the direct communications between pulmonary and systemic circulations, bypassing the capillary bed, which leads to physiological abnormalities, in particular right-to-left shunts which cause hypoxaemia with subsequent paradoxical embolism. ${ }^{[5]}$

PAVMs may be single or multiple, unilateral or bilateral, and simple or complex. Most PAVMs are congenital with acquired causes including conditions such as post-thoracic surgery, trauma, tuberculosis, actinomycosis and schistosomiasis. ${ }^{[2,3]} 53-70 \%$ of PAVMs are found in lower lobes with a preponderance of unilateral disease ${ }^{[3]}$ Clinically, patients present most commonly with epistaxis, dyspnoea, sometimes platypnoea and haemoptysis, but may be asymptomatic. Less common complaints include cough, chest pain, migraine headaches, tinnitus, dizziness, dysarthria, syncope, vertigo and diplopia. Signs commonly found include bruit, clubbing, cyanosis and telangiectasia. ${ }^{[2]}$

\section{Conclusion}

The clinical features of PAVMs resemble many respiratory conditions, a chest radiograph is thus mandatory to diagnosis PAVMs with CT or pulmonary angiography being diagnostic. Chest radiography is an important diagnostic tool and reveals abnormalities in $98 \%$ of patients. ${ }^{[3]}$ Classic finding on chest radiograph is that of a round or oval mass of uniform density, often lobulated but sharply defined, more commonly in lower lobes and ranging from 1 to $5 \mathrm{~cm}$ in diameter. ${ }^{[2]}$ However, chest $\mathrm{CT}$ is more accurate in identifying connecting vessels and PAVM. The diagnosis of PAVM should be suspected with any of the following presentations: (a) classic findings on chest radiograph as described, (b) mucocutaneous telangiectasis and (c) unexplained findings such as dyspnoea, haemoptysis, hypoxaemia, polycythaemia, clubbing, cyanosis, cerebral embolism or brain abscess. ${ }^{[2]}$ Advances in endovascular techniques have made embolotherapy the preferred treatment option which reduces the need for surgical intervention. The practice of careful and precise angiography techniques utilising modern coaxial catheters, coils and other embolic devices, means almost all PAVMs can be successfully treated. ${ }^{[2]}$

\section{Key learning points}

- PAVMs are rare pulmonary vascular anomalies, with more than $80 \%$ being congenital.

- PAVMs can cause dyspnoea with right-to-left shunts, commonly with cyanosis, clubbing and pulmonary vascular bruit.

- Because of paradoxical emboli, patients can present with central nervous system complications.

- Chest radiography and contrast CT are essential diagnostic tools, but pulmonary angiography is the gold standard.

- Treatment options include angiography techniques utilising modern coaxial catheters, coils and other embolic devices.

- Advanced treatment modalities means almost all PAVMs can be successfully treated.

\section{References}

1. MeekME, Meek JC, Beheshti MV. Management of pulmonary arteriovenous malformations. Semin Intervent Radiol 2011;28(1):24-31.[http://dx.doi.org/10.1055/s-0031-1273937]

2. Gossage JR, Kanj G. Pulmonary arteriovenous malformations: A state of the art review. Am J Respir Crit Care Med 1998;158(2):643-661.[http://dx.doi.org/10.1164/ajrccm.158.2.9711041]

3. Bandyopadhyay SK, Nandy A, Sarkar S, Ghosal AG. Massive haemothorax: A presentation of pulmonary arteriovenous malformation. Indian J Chest Dis Allied Sci 2008;50(3):285-287.

4. Todo K, Moriwaki H, Higashi M, Kimura K, Naritomi H. A small pulmonary arteriovenous malformation as a cause of recurrent brain embolism. AJNR Am J Neuroradiol 2004;25(3):428-430.

5. Shovlin CS, Letarte M. Hereditary haemorrhagic telangiectasia and pulmonary arteriovenous malformations: Issues in clinical management and review of pathogenic mechanisms. Thorax 1999;54(8):714-729.

\title{
Superior mediastinal masses in children - two cases of lymphoma
}

\author{
A C Jeevarathnum, MB BCh, FCPaed (SA), Dip Allergy (SA), MMed, Cert Paed Pulm (SA), European Respiratory Diploma; \\ A van Niekerk, MB BCh, MMed; D Parris, BSc, MB BCh, FCPaed (SA), Dip Allergy (SA); K De Campos, MB ChB, MMed, Dip Allergy (SA); \\ W Wijnant, MD Paed, Dip Allergy (SA), Cert Paed Pulm(SA); X Deadren, MB ChB, FCPaed (SA), MMed;
}

A Büchner, MB ChB, DCH (SA), FCPaed(SA), MMed, Dip Pall Med, Cert Med Oncol (Paed)(SA);

F Omar, MB ChB, FCPaed (SA), Cert Paed Med, Onc Paed (SA);

D Reynders, MB ChB, FCPaed (SA), MRCPCH, Cert Paed Med, Onc Paed (SA); R J Green, PhD, DSc

Department of Paediatrics and Child Health, School of Medicine, Faculty of Health Sciences, University of Pretoria and Steve Biko Academic Hospital, Pretoria, South Africa

Corresponding author: A C Jeevarathnum (acjeevarathnum@gmail.com)

The exact incidence of superior mediastinal masses in children is largely unknown. They present as a spectrum of disease ranging from an incidental finding on a chest X-ray to being markedly symptomatic with superior vena caval syndrome or obstruction of the upper airways. Lymphomas are the most common causes of superior mediastinal masses in children. We present two cases of confirmed T-cell lymphoma 
in children with superior mediastinal masses. In doing so, we explore a diagnostic approach and visit the complications the physician needs to be aware of when confronted with a child with a superior mediastinal mass.

S Afr Respir J 2016;22(1):23-25. DOI: 10.7196/SARJ.2016.v22i1.58

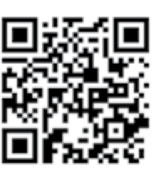

\section{Case 1}

The first patient is a 4-yearold HIV-negative male who presented to Steve Biko Academic Hospital with a 1-week history of cough and shortness of breath. This was the first time this child had been ill. There was no other significant history and there were no tuberculosis (TB) contacts.

Clinically this child was in moderate respiratory distress and was oxygen dependent. There was shift of the mediastinum to the right and stony dullness to percussion on the left, indicative of a left-sided pleural effusion. There were no significant peripheral nodes that could be biopsied and there was no hepatosplenomegaly.

A frontal chest X-ray (CXR) of this child confirmed a left-sided pleural effusion and a widened superior mediastinum as evidenced by Fig. 1 . The widened superior mediastinum became more apparent on drainage of the effusion. The computed tomography (CT) confirmed the presence of a large superior mediastinal mass as in Fig. 2. The effusion was exudative in nature with a very high adenosine deaminase level of $184 \mathrm{U} / \mathrm{L}$. Cytology of the effusion revealed atypical lymphocytes suggestive of a malignancy. The child's white cell count was normal with no atypical lymphocytes on smear. Tumour markers including serum lactate dehydrogenase (LDH) were not elevated.

The superior medistinal mass was biopsied by the cardiothoracics surgery team and a bone marrow aspirate and trephine (BMAT) was performed concurrently. The anaesthetic for the entire process was conducted in

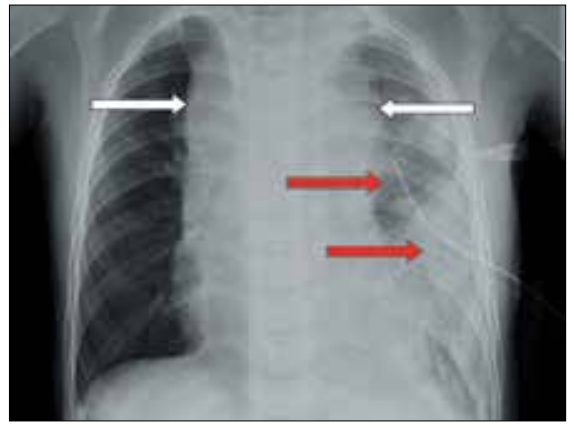

Fig 1. Frontal chest $X$-ray of patient 1 revealing a widened superior mediastinum (white arrows) and an intercostal drain on the left (red arrows). an extremely cautious fashion with a gas induction and with spontaneous respiration via endotracheal tube during the procedure. The BMAT was not suggestive of malignancy. Histology of the mass itself revealed a T-cell lymphoblastic lymphoma as depicted in Fig. 3.

\section{Case 2}

The second case was a 2-year-old HIV-negative male who presented with an acute history of cough and shortness of breath following a choking episode. Considering the history, a foreign body was the initial concern. This was a clinically well child with no respiratory symptomatology. The frontal CXR revealed an incidental finding of a widened superior mediastinum as depicted in Fig. 4. A contrasted CT scan of the chest confirmed a homogenous superior mediastinal mass in the anterior compartment, as shown in Figs. 5. There were no calcifications or cystic changes that would suggest a teratoma. The CT did reveal evidence of compression of the trachea although the child was clinically asymptomatic. In this case as well, there were no peripheral lymph nodes to biopsy and haematological workup including tumour markers was non-contributory. On awaiting theatre for a histological specimen of the mass, the patient had an unexpected cardiorespiratory arrest and unfortunately died.

A postmortem examination revealed no evidence of a foreign body aspiration as suggested by the history. Histology of the mass revealed a T-cell lymphoma. The cause of death was most likely upper airway obstruction from a very large tumour.

Neither patient received corticosteroids while awaiting theatre.

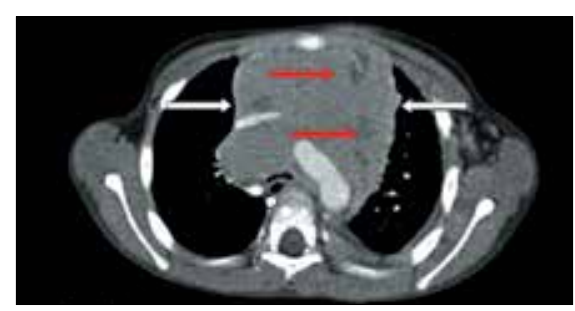

Fig. 2. Axial contrasted CT)at the level of the carina demonstrating the large superior mediastinal mass (white arrows) with areas of necrosis and cystic change (red arrows).

\section{Discussion}

Depending on the compartment of the mediastinum involved, there are a number of causes of a widened mediastinum, as shown in Table $1 .^{[1-3]}$ This is imperative in trying to define the aetiology. The majority of mediastinal masses in children are malignant. ${ }^{[2]}$

Lymphomas are the most common cause of mediastinal masses in the paediatric population. ${ }^{[1,4]}$ Between 50 and $70 \%$ of patients with lymphoblastic lymphomas present with an anterior mediastinal or intrathoracic mass. ${ }^{[1]}$ In the paediatric population, two-thirds of lymphomas occurring in the mediastinum are nonHodgkin's lymphoma, and the remainder are Hodgkin's lymphoma. The second most common cause of mediastinal masses in the anterior mediastinum are germ cell tumours including benign teratomas in addition to malignant seminomas and yolk sac tumours. Germ cell tumours peak in incidence at 3 years of age and at adolescence. The mediastinum is the fourth most common site for teratomas. Neurogenic tumours including neuroblastoma are the most common causes of posterior mediastinal masses.

The diagnostic evaluation begins with a frontal and lateral chest X-ray in which $90 \%$ of mediastinal masses can be seen. ${ }^{[2]}$ A CT scan of the chest is necessary to anatomically define the extent and nature of the mass, define the compartment of the mediastinum in which the mass occurs and to determine the degree of airway compression. ${ }^{[2]}$ There are certain clues that could point to a specific diagnosis with fat, fluid and calcified components being more common in germ

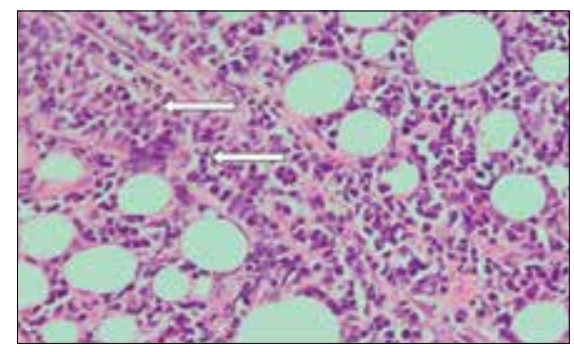

Fig. 3. H\&E section of the mass seen in figures revealing extensive lymphocyte proliferation (courtesy of Dr J Dinkel, Department of Anatomical Pathology, Tshwane Academic Division, NHLS). 


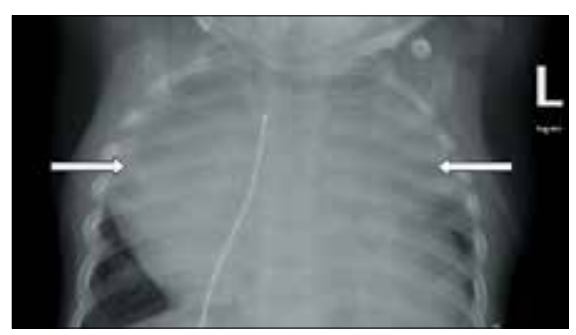

Fig. 4. Frontal chest $X$-ray of patient 2 demonstrating large superior mediastinal mass (arrows).

cell tumours. ${ }^{[3,5]}$ The involved compartment will guide the differential diagnosis. ${ }^{[5]}$

Haematological markers that are useful include a full blood count with differential and (looking for atypical lymphocytes or blasts, or cytopenias indicating possible bone marrow infiltration) tumour markers (alpha fetoprotein, beta human chorionic gonadotrophin (HCG)) in the case of germ cell tumours. A BMAT is necessary in the case of a suspected haematological malignancy, as one-third of patients will have bone marrow involvement. ${ }^{[2]}$ A biopsy of the mass itself is mandatory in those cases where a diagnosis cannot be made with peripheral specimens (biopsy of peripheral lymph nodes or other blood investigations). Biopsy of the mass itself can be obtained by a CT-guided procedure or via sternotomy. Certain tumour markers may assist with a diagnosis: for instance, elevated alpha foetoprotein and beta HCG would suggest a germ cell tumour. ${ }^{[5]} \mathrm{A}$ peripheral flow cytometry conducted on a patient with a very high white cell count would suggest a lymphoma/leukaemia. Elevated urinary levels of the catecholamine vanillylmandelic acid (VMA) and homovanillic acid (HVA) in a patient with a posterior mediastinal mass is suggestive of a neuroblastoma. ${ }^{[6]}$

When planning a biopsy, the clinician needs to be aware that there is a significant anaesthetic risk with any form of sedation and induction of anaesthesia leading to possible acute airway obstruction, sudden cardiac arrest and death. ${ }^{[4,-9]}$ The risk is present even in the case of an asymptomatic lesion. ${ }^{[4]}$ Children with superior mediastinal masses are at higher risk of an anaesthetic death than their adult counterparts. ${ }^{[9]}$ Conscious sedation with spontaneous breathing during the procedure is the recommended method of anaesthesia in these patients and neuromuscular blockade is not advised. ${ }^{[4,8,9]}$

The role of preoperative steroids needs to be clearly defined, the advantage of which will decrease the risk of airway obstruction

Table 1. Mediastinal masses by location

\begin{tabular}{lll}
\hline Anterior & Middle & Posterior \\
\hline Non-Hodgkin's lymphoma & Vascular malformations & Neuroblastoma \\
Hodgkin's lymphoma & Double aortic arch & Ganglioneuroblastoma \\
Germ cell tumour & Pulmonary artery sling & Ganglioneuroma \\
Hyperplastic/ectopic thymus & Aneurysms & Nerve sheath tumours \\
Thymoma & Bronchogenic/foregut cysts & \\
& Mediastinal lymph nodes &
\end{tabular}
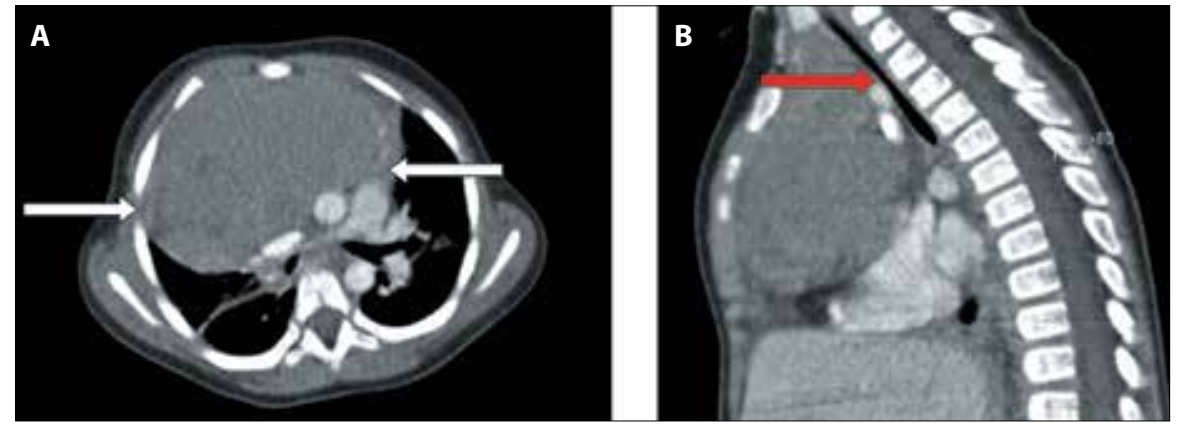

Fig. 5A and B. Axial (A) and sagittal (B) CT views of patient 2 revealing a large homogenous superior mediastinal mass (white arrows). Tracheal compression is noted in sagittal view (red arrow).

by shrinking the tumour and improving the anaesthetic outcome. ${ }^{[10]}$ However, this runs the risk of an inadequate biopsy specimen and could potentially interfere with a histological diagnosis. ${ }^{[7]}$ In one series of 18 patients, preoperative steroids were used in patients with features of airway compromise and despite this a good histological sample was obtained in $95 \%$ of cases; prolonged use of steroids ( $>5$ days) impaired histological diagnosis in $5 \%$ of cases. ${ }^{[7]}$ Another series also concluded minimal interference with pathological diagnosis with the use of preoperative steroids in high-risk patients. ${ }^{[10]}$ This is definitely an area that needs further research and exploration.

In case 2, starting preoperative steroids would have possibly resulted in tumour shrinkage and avoided an unfortunate demise. This is definitely a learning point in the case.

\section{Conclusion}

Lymphomas are one of the most common causes of superior mediastinal masses in the paediatric population. A mass in the superior mediastinum usually requires a histological diagnosis. These patients, despite appearing clinically stable, can be challenging to manage and caution should be employed during the time of biopsy when anaesthetic is administered. The physician should consider the use of preoperative steroids in symptomatic individuals or asymptomatic individuals with evidence of airway compression on imaging in order to improve the anaesthetic outcome.

\section{References}

1. Williams HJ, Alton HM. Imaging of paediatric mediastinal abnormalities. Paediatr Respir Rev 2003;4(1):55-66. [http://dx.doi.org/10.1016/S15260542(02)00310-X]

2. Jaggers J, Balsara K. Mediastinal masses in children. Sem Thorac Cardiovasc Surg 2004;16(3):201-208. [http://dx.doi.org/10.1053/j.semtcvs.2004.08.005]

3. Ranganath SH,LeeEY, Restrepo R, Eisenberg RL. Mediastinal masses in children. ARJ Am J Roentgenol 2012;198(3):197216. [http://dx.doi.org/10.2214/AJR.11.7027]

4. Suominen PK, Kanerva JA, Saliba KJ, Taivainen TR. Unrecognised mediastinal tumor causing sudden tracheal obstruction and out-of-hospital cardiac arrest. J Emerg Med 2010;38(5):e63-e66. [http:// dx.doi.org/10.1016/j.jemermed.2007.10.065]

5. Kennebeck SS. Tumours of the mediastinum. Clin Ped Emerg Med 2005;6:156-164. [http://dx.doi. org/10.1016/j.cpem.2005.05.003]

6. Kushner BH. Neuroblastoma: A disease requiring a multitude of imaging studies. J Nucl Med 2004;45(7):1172-1188.

7. Hack HA, Wright NB, Wynn RF. The anaesthetic management of children with anterior mediastinal masses. Anaesthesia 2008;63(8):837-846. [http:// dx.doi.org/10.1111/j.1365-2044.2008.05515.x]

8. Gothard JW. Anaesthetic considerations for patients with anterior mediastinal masses. Anesthesiol Clin 2008;26:305-314. [http://dx.doi.org/10.1016/j. anclin.2008.01.002]

9. Slinger P, Karsli C. Management of the patient with a large anterior mediastinal mass: Recurring myths. Curr Opin Anaesthesiol 2007;20(1):1-3.

10. Borenstein SH, Gerstle T, Malkin D, Thorner P, Filler RM. The effects of prebiopsy corticosteroid treatment on the diagnosis of mediastinal lymphoma. J Pediatr Surg 2000;35(6):973-976. [http://dx.doi.org/10.1053/jpsu.2000.6945] 